\title{
EL ESTADO ACTUAL DE LA EDUCACIÓN MANABITA
}

\author{
CURRENT STATUS OF EDUCATION MANABITA
}

\author{
Ing. Civil. Fabrina Monserrate Cedeño Mendoza \\ fcedeno@itsup.edu.ec \\ INSTITUTO TECNOLÓGICO SUPERIOR PORTOVIEJO “ITSUP”, ECUADOR
}

\section{RESUMEN}

El presente artículo me permite publicar, el desarrollo que ha tenido la educación manabita a través de los años; así, como el docente que refleja experiencias, dominio de técnicas, autoconfianza, la evolución del sistema educativo y, el proceso de evaluación que ayuda a mejorar la calidad y detectar los puntos fuertes y débiles de la institución a la que pertenece el docente, todos estos aspectos se reflejan en los resultados obtenidos con más relevancia en la presente investigación. No obstante la preparación de sus recursos humanos, hace que la educación manabita tenga un papel cada vez más importante en la sociedad. Y es que la evolución educativa se ha dejado ver por todos y todas que de una forma desinteresada pretenden en los estudiantes brindar una educación de buena calidad.

Palabras claves: autoconfianza; educación; evaluación; experiencia docente; proceso

\section{SUMMARY}

This article allows me to publish, the development that has taken manabita education through the years, as well as the teacher reflecting experiences, mastery of skills, selfconfidence, the evolution of the educational system and the evaluation process that helps improve quality and identify strengths and weaknesses of the institution to which the teacher belongs, all these aspects are reflected in the results more relevant to the present investigation. However the preparation of human resources, education makes manabita have a increasingly important role in society. And the educational development has been allowed to see everyone in a selfless that aim to provide students quality education.

Keywords: self-confidence, education, assessment, teaching experience, process 


\section{INTRODUCCIÓN}

El estudio que ostenta este artículo se enmarca dentro de un proyecto de investigación de análisis social-educativo denominado "EL ESTADO ACTUAL DE LA EDUCACIÓN MANABITA". Se trata de un proyecto de carácter personal con el soporte facultativo de los docentes con nombramiento, que visitaron de forma esporádica la COORDINACIÓN ZONAL N 4 DE EDUCACIÓN, Manabí - Santo Domingo de los Tsáchilas

Es notoria la evolución educativa en la provincia de Manabí, y es que ha sido un largo e incesante proceso y como tal no debería permitir interrupciones abruptas. Su continuidad se sustenta en los docentes con la condición de que transmitan su experiencia depurada por los logros obtenidos, lo que permite que los que vienen detrás sean más eficaces pues se les abrevia el tiempo para alcanzar la madurez profesional.

El Ecuador y por ende la provincia de Manabí vive un período de amplias realizaciones y cambios, pero, lo que es más importante, de crecientes preocupaciones respecto de la enseñanza que se brinda. La marcha educativa padeció siempre de fallas, esto se produjo al no haber compactado la proyección educativa con la realidad socio económico que se vive. Uno de los problemas que la educación manabita quiere eliminar es el analfabetismo, el cual, si bien ha disminuido en los últimos años sigue siendo alto. 


\section{FUNDAMENTACIÓN}

Katarina Tomasevski valora, el avance parcial registrado durante las últimas décadas en el ámbito educativo, y que "todo el mundo asuma ya que la educación tiene que ser gratuita". Destaca que "sin dinero, ningún objetivo es posible, pero sólo con dinero no se alcanzará si cada gobierno no se compromete con la educación", y citó como ejemplo el caso de Pakistán, que cuenta con ayudas para la educación y sin embargo la inversión en esta materia no alcanza el 1\% de su Producto Interno Bruto (PIB).

La relatora especial de la Organización de las Naciones Unidas (ONU), experta independiente, menciona que entre los objetivos del milenio que se ha fijado la ONU figura el de garantizar la educación gratuita para todos los niños en el año 2015, y advierte que muchos países "no pueden esperar tanto tiempo". Aún así, Katarina Tomasevski se define como "una optimista incurable", y señala que "todos los logros en materia de derechos humanos se han conseguido cuando la sociedad se ha movilizado".

Cabe señalar que el Gobierno Nacional de la República del Ecuador está reorganizando la educación técnica en el país, en donde existe el bachillerato unificado técnico, con materias extras, como agricultura o metalmecánica, así mismo el post bachillerato de formación técnica el cual durará dos años y los institutos tecnológicos, quienes son los encargados de entregar títulos técnicos; el 
desafío del Gobierno de Ecuador es llegar al 23\% de formación técnica hasta el 2016, que es el promedio de la mayoría de países desarrollados.

En cuanto a la educación inicial, el Gobierno Nacional, a través del Ministerio del ramo, está creando 140 distritos, que serán una nueva forma de brindar servicios a los estudiantes, a los padres de familia y a los docentes, entre ellos destacan: régimen escolar, denuncias, asignación de alimentación, entre otros.

\section{MÉTODO}

\section{Participantes}

La muestra de este estudio ha estado compuesta por 80 docentes con nombramiento, que visitaron de forma esporádica la COORDINACIÓN ZONAL Nº 4 DE EDUCACIÓN, Manabí - Santo Domingo de los Tsáchilas, durante el mes de abril de 2013.

\section{INDICADORES DE LA ENCUESTA}

1.- Marque aquellos factores con los que usted considere que la educación en la provincia de Manabí ha evolucionado. 


\begin{tabular}{|c|l|c|c|}
\hline Orden & \multicolumn{1}{|c|}{ Alternativas } & f & \% \\
\hline a) & Bajo nivel de escolaridad & 9 & 9 \\
\hline b) & Persistencia del analfabetismo & 8 & 8 \\
\hline c) & Mala infraestructura de los establecimientos & 5 & 5 \\
\hline d) & Falta de materiales didácticos & 3 & 3 \\
\hline e) & Elevada tasa de repetición & 9 & 9 \\
\hline f) & Todas las anteriores & 66 & 66 \\
\hline & \multicolumn{1}{|c|}{ TOTAL } & $\mathbf{1 0 0}$ & $\mathbf{1 0 0}$ \\
\hline
\end{tabular}

Del total de la muestra, el $66 \%$ considera que la educación en la provincia de Manabí ha evolucionado notoriamente; a diferencia de un 33\% que se manifiesta indicando que sólo un $9 \%$ ha evolucionado el bajo nivel de escolaridad, un $8 \%$ ha evolucionado en la persistencia del analfabetismo, un $5 \%$ menciona que ha evolucionado la mala infraestructura de los establecimientos, tan solo un $3 \%$ indica que ha evolucionado en la falta de materiales y el $9 \%$ restante menciona que ha evolucionado la elevada tasa de repetición. Según los indicadores que establece la Organización de las Naciones Unidades para la Educación, la Ciencia y la Cultura (UNESCO) en septiembre de 2011 declara a Santa Ana de Vuelta Larga de la Provincia de Manabí como el primer cantón libre de analfabetismo. La encuesta se ve reflejada en el gráfico $\mathrm{n}$ ㅇ 1.

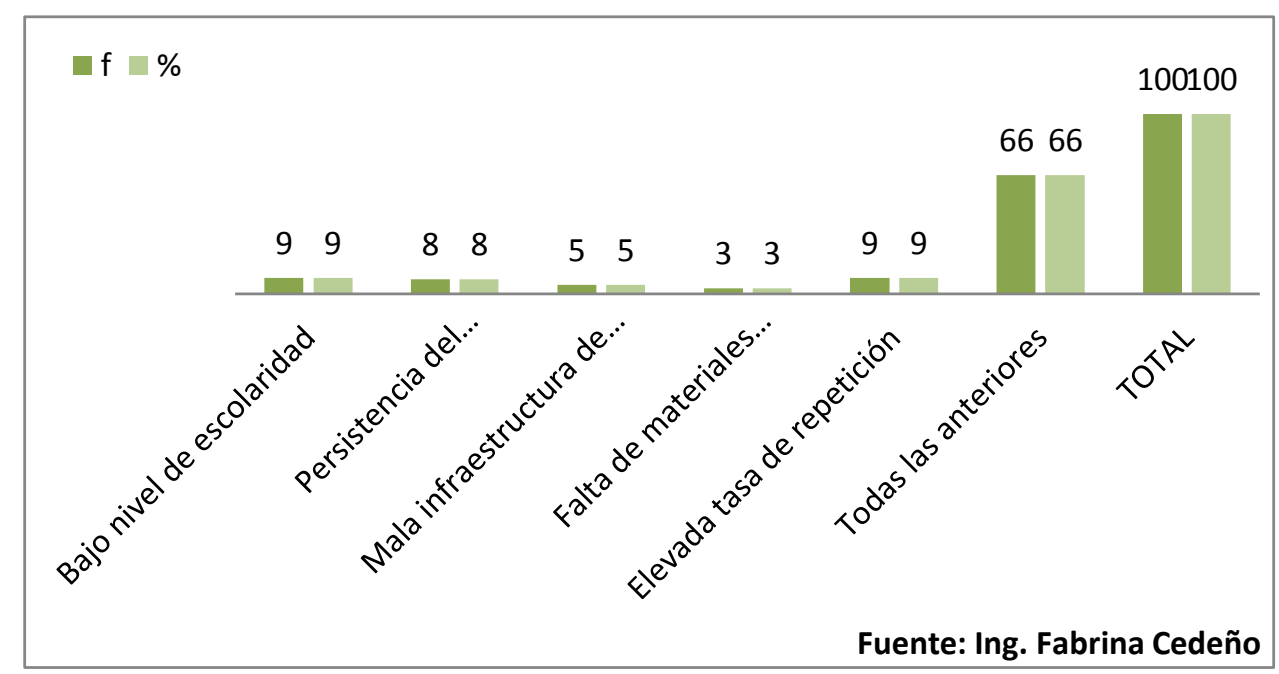


2. Cree usted que el docente ejerce la profesión por los siguientes factores.

\begin{tabular}{|c|l|c|c|}
\hline Orden & Alternativas & f & $\mathbf{\%}$ \\
\hline a) & Rutina & 18 & 18,95 \\
\hline b) & Sueldo & 23 & 24,21 \\
\hline c) & Enseñar & 54 & 56,84 \\
\hline & & $\mathbf{1 0 0}$ & $\mathbf{1 0 0}$ \\
\hline
\end{tabular}

Del total de la muestra, el 56,84 \% considera que el docente ejerce la profesión para enseñar; pero un 18,95\% menciona que el docente ejerce la profesión por rutina y un $24,21 \%$ se manifestó indicando que el docente ejerce su profesión por el sueldo. Lamentablemente hay algunos docentes, que ejercen esta profesión sin vocación, y por lo tanto, los gana la rutina, el desgano; y cada año o semestre que pasa, en lugar de enriquecerse con su experiencia, ésta se le viene encima, abatiéndolos, y solo esperan que llegue el mensual, el décimo o la jubilación, contagiando esta abulia a los/as estudiantes. La encuesta se ve reflejada en el gráfico $\mathrm{n}$ ㅇ 2 .

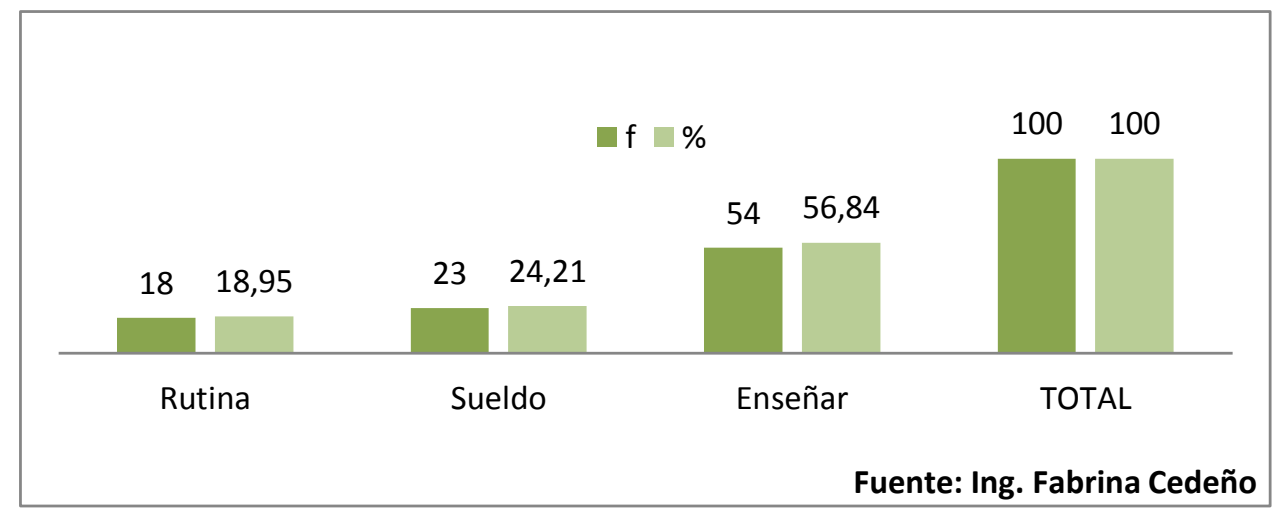


3. Marque los aspectos que usted considere que debe ser capaz de expresar y reflejar un docente en el aula de clases.

\begin{tabular}{|c|l|c|c|}
\hline Orden & \multicolumn{1}{|c|}{ Alternativas } & f & $\%$ \\
\hline a) & Transmitir experiencia & 2 & 2,4 \\
\hline b) & Contagiar actitudes de respeto & 2 & 2,4 \\
\hline c) & Manifestaciones de entusiasmo y calidez & 1 & 1,2 \\
\hline d) & Autoconfianza & 3 & 3,6 \\
\hline e) & Dominio de técnicas y recursos & 5 & 6,1 \\
\hline f) & Todas las anteriores TOTAL & 70 & 84,3 \\
\hline & $\mathbf{8 3}$ & $\mathbf{1 0 0}$ \\
\hline
\end{tabular}

Del total de la muestra, un $84,3 \%$ considera que el docente debe ser capaz de expresar y reflejar en el aula de clases las alternativas que se presentan en la encuesta; a diferencia de un porcentaje mínimo como es el $2,4 \%$ en el que debe transmitir experiencia, un 2,4\% corresponde contagiar actitudes de respeto, el 1,2\% conviene manifestar entusiasmo y calidez, en cambio el $3,6 \%$ se obliga desarrollar autoconfianza, y el 6,1\% restante manifiesta que el docente debe tener dominio de técnicas y recursos en el aula de clases. Una condición fundamental del buen docente es su compromiso con la formación humana. La posibilidad de formar exige al docente un proyecto de vida consecuente con los principios que orientan su labor educativa. La encuesta se ve reflejada en el gráfico № 3. 


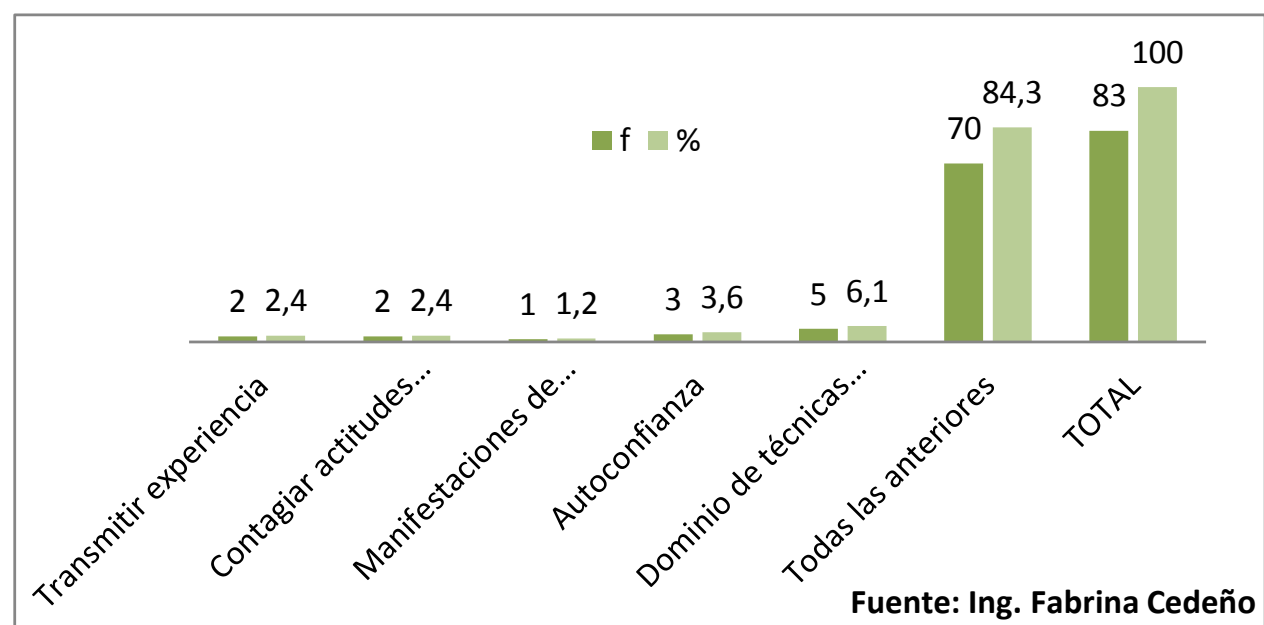

4.

Considera usted que en el centro educativo al que pertenece, el área administrativa hace gestiones para elevar el nivel académico.

\begin{tabular}{|c|c|c|c|}
\hline Orden & Alternativas & $f$ & $\%$ \\
\hline a) & Totalmente & 24 & 30 \\
\hline b) & Parcialmente & 14 & 17,5 \\
\hline c) & De ninguna manera & 42 & 52,5 \\
\hline & TOTAL & 80 & 100 \\
\hline
\end{tabular}

Del total de la muestra, el 52,5\% considera que el área administrativa no realiza gestiones para elevar el nivel académico, en cambio un $30 \%$ considera que totalmente y parcialmente en un $17,5 \%$. Para que haya una buena Calidad Educativa, lo primero que debe haber es una buena Gestión Administrativa. La encuesta se ve reflejada en el gráfico no 4 .

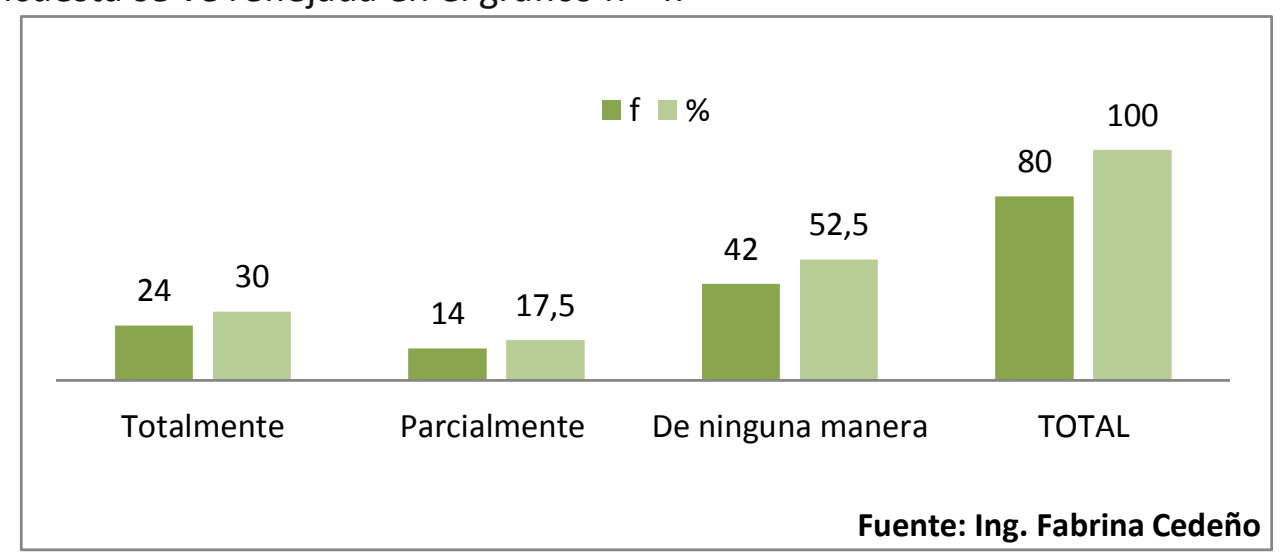


5. Marque aquellas fases que usted considera necesarias del sistema educativo.

\begin{tabular}{|c|l|c|c|}
\hline Orden & \multicolumn{1}{|c|}{ Alternativas } & f & \% \\
\hline a) & Conocimiento del entorno & 4 & 4,5 \\
\hline b) & Conocimiento del potencial de la institución & 3 & 3,4 \\
\hline c) & Armonización de objetivos & 5 & 5,6 \\
\hline d) & Elección del procedimiento & 2 & 2,2 \\
\hline e) & Conducción del sistema & 0 & 0 \\
\hline f) & Ejecución y control & 5 & 5,6 \\
\hline g) & Todas las anteriores TOTAL & $\mathbf{7 0}$ & $\mathbf{7 8 , 7}$ \\
\hline & $\mathbf{8 9}$ & $\mathbf{1 0 0}$ \\
\hline
\end{tabular}

Del total de la muestra, el $78,7 \%$ considera que todas las fases mencionadas en la encuesta son necesarias en el sistema educativo, y de forma parcial considera que un $4,5 \%$ sobre el conocimiento del entorno, un $3,4 \%$ sobre el conocimiento del potencial de la institución, el 5,6\% la armonización de objetivos, un 2,2\% en la elección del procedimiento y el $5,6 \%$ en la ejecución y control. Un sistema

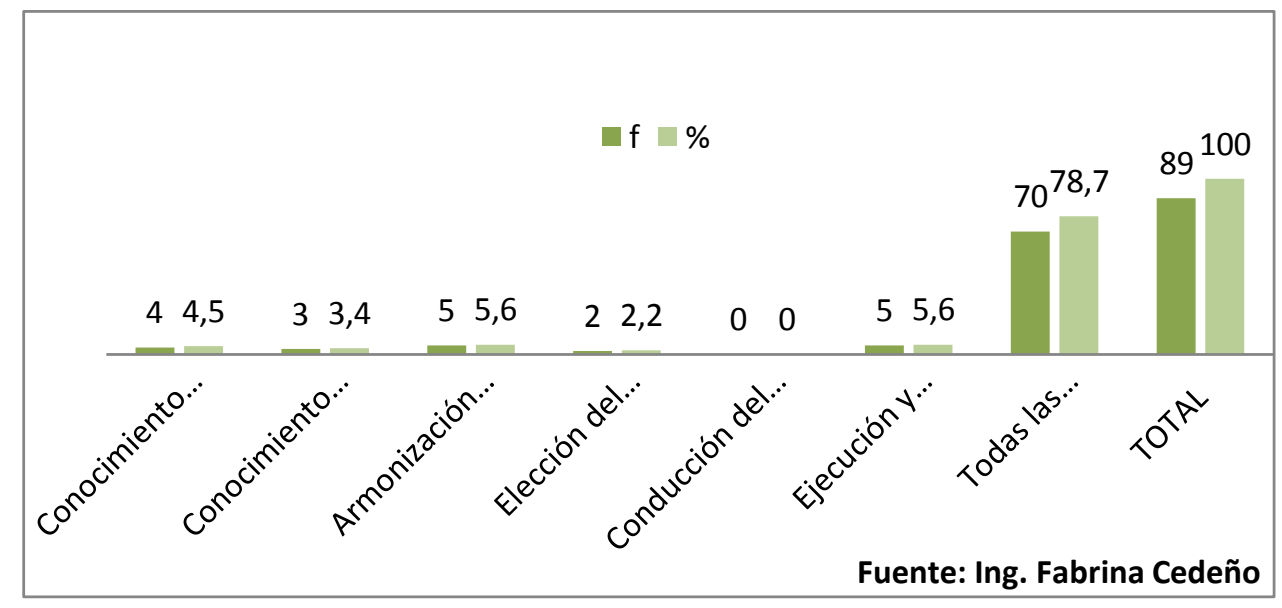


educativo que no considere todas las fases está evadiendo la enseñanza, seria y profunda para formar individuos eminentes; en estas condiciones jamás será una educación de buena calidad, y cuando a esta se suma una falta de enseñanza de los mismos valores dentro del hogar la catástrofe es ineludible. La encuesta se ve reflejada en el gráfico $n$ 으. 5 .

6. Considera usted que el proceso de evaluación ayuda a mejorar la calidad y detectar los puntos fuertes y débiles de su institución.

\begin{tabular}{|c|l|c|c|}
\hline Orden & \multicolumn{1}{|c|}{ Alternativas } & F & \% \\
\hline a) & Definitivamente si & 73 & 91,25 \\
\hline b) & Probablemente si & 4 & 5 \\
\hline c) & Indeciso & 0 & 0 \\
\hline d) & Probablemente no & 3 & 3,75 \\
\hline e) & Definitivamente no & 0 & 0 \\
\hline & TOTAL & $\mathbf{8 0}$ & $\mathbf{1 0 0}$ \\
\hline
\end{tabular}

Del total de la muestra, el 91,25\% considera que el proceso de evaluación ayuda a mejorar la calidad y detectar los puntos fuertes y débiles de la institución, un 5\% probablemente sí y un 3,75\% probablemente no. Se expone el proceso de evaluación como una particularidad para iniciar los procesos de mejoramiento y autorregulación institucional, juzgando sus ventajas pero también planteando sus

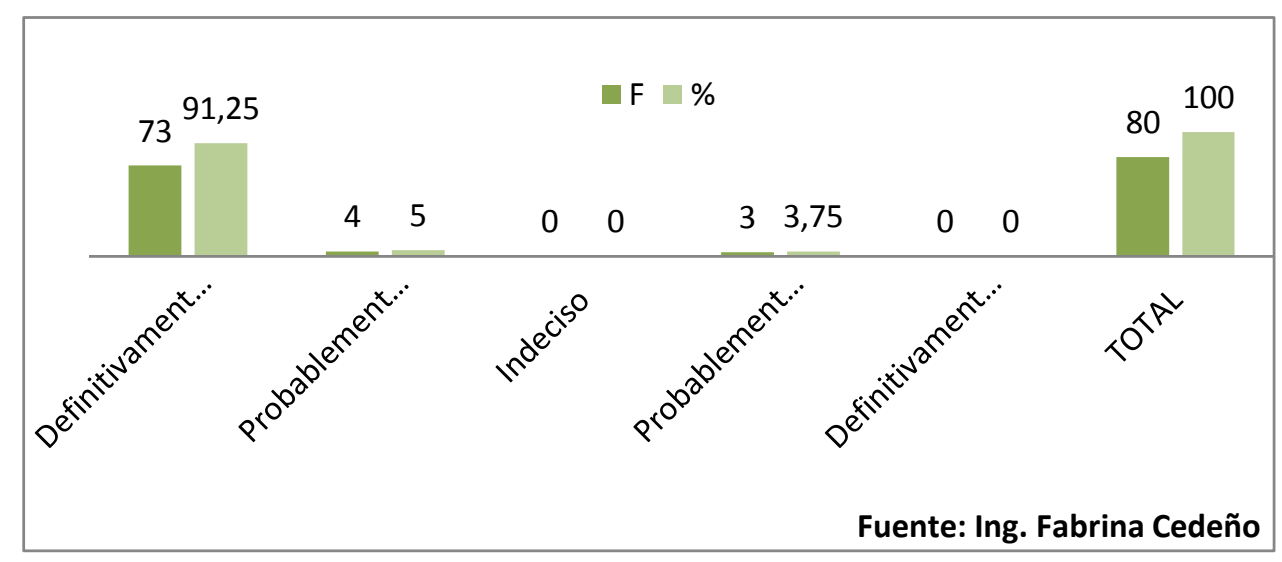


paradigmas y limitaciones, ya que cualquier bien pensado y diseñado proceso podría tener cierto grado de dificultad y firmeza en la etapa de ejecución; es por ello la necesidad de argumentar en forma razonada los desafíos más relevantes.

\section{DISEÑO Y PROCEDIMIENTO}

El trabajo de campo fue realizado bajo mi dirección y responsabilidad directa con la colaboración de 80 docentes con nombramiento, que visitaron de forma esporádica la COORDINACIÓN ZONAL Nº 4 DE EDUCACIÓN, Manabí - Santo Domingo de los Tsáchilas, durante el mes de abril de 2013, la idea surge de conocer el estado actual de la educación manabita, debido a los grandes cambios que se están emprendiendo para transformar radicalmente la educación en la provincia de Manabí; sean estos a nivel de estructura, material didáctico, docentes mejores capacitados, con técnicas apropiadas para enfrentar el nuevo sistema educativo; el cual se desarrolló en el mes de abril. Previamente, durante el mes de marzo se diseñó la encuesta y se planificaron todas las fases del desarrollo del proyecto. A finales de abril se procedió a efectuar la encuesta y, la primera semana de mayo se realizó el tratamiento de análisis de los datos obtenidos, empleando para ello tabulaciones y gráfico de barras.

\section{DISCUSIÓN Y CONCLUSIONES}

Los resultados obtenidos en esta investigación sobre "EL ESTADO ACTUAL DE LA EDUCACIÓN MANABITA" reflejan que, la educación ha evolucionado 
notoriamente, así como lo indica la pregunta uno (1), además que el docente ejerce su profesión transmitiendo experiencia con respeto y autoconfianza y, finalmente consideran que el proceso de evaluación ayuda a mejorar la calidad y detectar los puntos fuertes y débiles de su institución. Estas evidencias han sido identificadas como relevantes para la actualidad de la educación manabita; y se destacan con mayores puntuaciones altas la pregunta tres (3) y la pregunta seis (6) (considerándolos de mayor importancia)

Considerando que la sociedad manabita vive momentos de cambio y oportunidades caracterizada por innovaciones de índole demográfica, económica, política y social. A partir de la ideología de que la educación debe ser componente clave para el desarrollo social, cultural, político y económico del país y para este caso de la provincia de Manabí; para el fortalecimiento de la soberanía nacional; para la construcción de una mejor inteligencia individual y colectiva; y para combatir eficazmente la pobreza, el propósito central es hacer de la educación manabita el gran proyecto nacional. Las personas son el recurso más valioso de una nación y es la educación el medio por excelencia para desarrollar sus capacidades y prepararlas para una vida plena y exitosa.

En el terreno educativo la provincia de Manabí actualmente afronta problemas diversos, entre ellos, los relacionados con la cobertura y la equidad que, pese a los esfuerzos de varias décadas, aún no se han resuelto satisfactoriamente. 
Además existen retos inmediatos e importantes que el desarrollo sustentable de la provincia de Manabí plantea al sistema educativo, entre otros: ampliar y diversificar aún más la oferta educativa de buena calidad en todos sus tipos, niveles y modalidades y acercarla a los grupos más desfavorecidos; y que la escolaridad contribuya a la formación integral de una ciudadanía madura, preparada para convivir en un país democrático y multicultural, en el terreno político y social. Además deberá responder oportunamente a las demandas de la sociedad del conocimiento.

En conclusión "EL ESTADO ACTUAL DE LA EDUCACIÓN MANABITA" establece un cambio notorio, pues ha mejorado en muchos ámbitos y, es que la evolución educativa se ha dejado ver por todos y todas que de una forma desinteresada pretenden en los estudiantes brindar una educación de buena calidad.

\section{REFERENCIAS BIBLIOGRÁFICAS}

http://comunidad-escolar.cnice.mec.es/743/info4.html Katarina Tomasevski, relatora especial de la ONU, presenta en Madrid su libro El asalto a la Educación

http://www.andes.info.ec/actualidad/7621.html

2002, EDITORIAL LIMUSA, S.A. DE C.V GRUPO NORIEGA EDITORES Balderas 95, mexico D.F. C.P. 06040 
ALANIS, A. (2000). Saber y saber hacer en la investigación. Revista digital de educación y nuevas tecnologías. Número 12 - Octubre 2000.

ALVAREZ, R. (1984). Universidad: Investigación y Productividad. Caracas. Ediciones Paral.

ARÉCHIGA, H. (1991). Universidad y Producción de Conocimientos. En Luis F. Bojalil (comp.). Universidad y Conocimiento. México. Universidad Autónoma Metropolitana Unidad Xochimilco.

BOJALIL, L. (1991). Universidad y Conocimiento. México. UNAM.

BUNGE, M. (1975). La Investigación Científica. Barcelona. Ariel.

BEHEZA, G. (1998). Reflexiones en torno a los principios básico de la investigación científica. En Revista Punto cero. Pp. 36-39.

DI PRISCO, C. Y WAGNER, Erika (1990). Investigación y Docencia en las Universidades. Caracas. Interciencia

RAMA, C. (2006). La evaluación: una nueva realidad universitaria en América Latina. UAPA - Universidad Abierta Para Adultos, 8

RUIZ, J. (2004). Cómo hacer una evaluación de centros educativos (Tercera ed.). Madrid, España: Narcea. 
SALGADO, F. (2011). La nueva institucionalidad de la educación superior en el Ecuador y los requerimientos para los programas de posgrado. Simposio Internacional sobre Acreditación de Programas de Posgrado. pp. 13. Quito: Universidad Andina Simón Bolívar.

VALENZUELA, R. (2011). Evaluación de instituciones educativas. México: Trillas http://www.ecuadorinmediato.com/index.php?module=Noticias\&func=news us er view\&id=5341\&umt=manabi espera mejoras en educacion

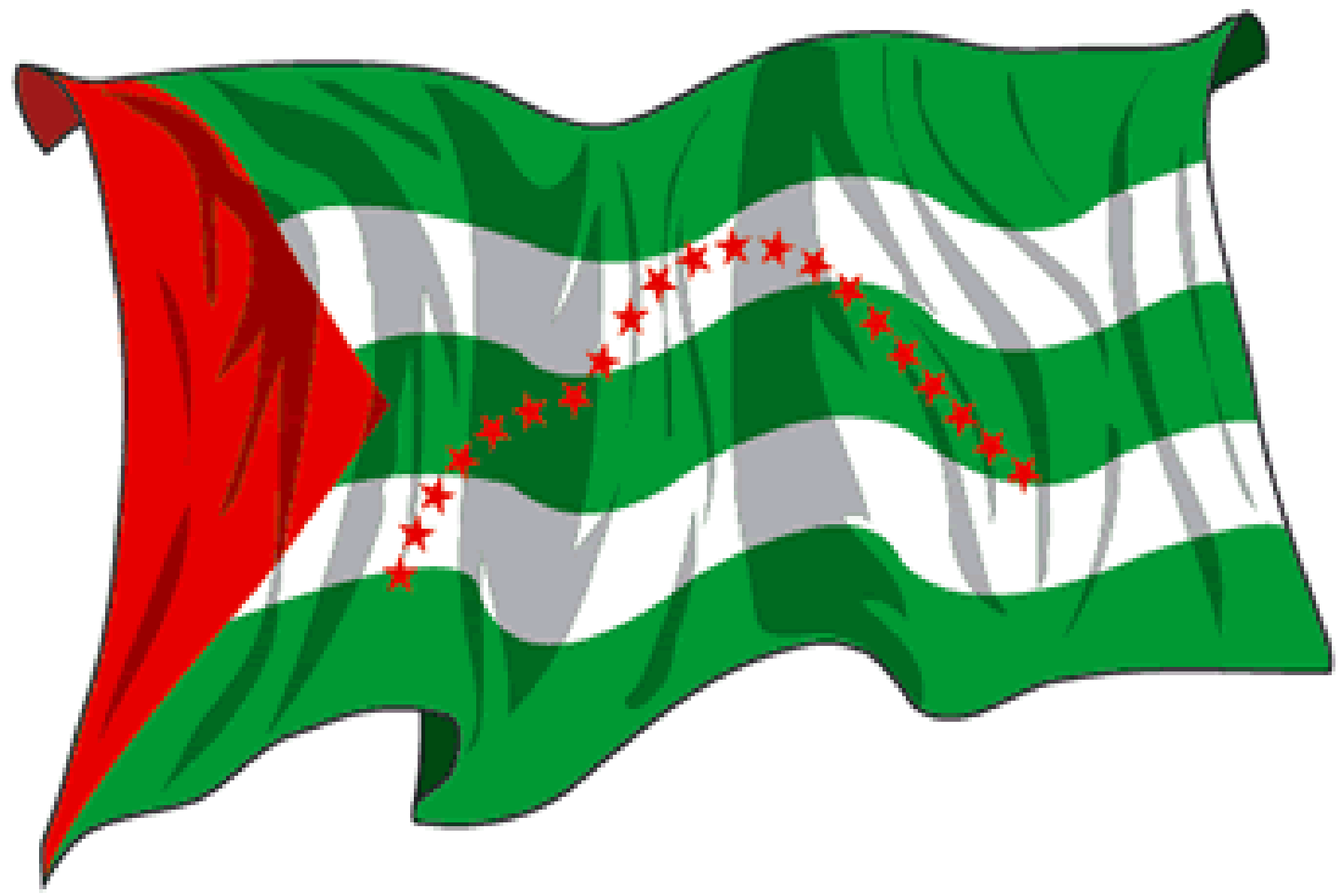

